\title{
MHD FLOW OF WALTERS' LIQUID B OVER A NONLINEARLY STRETCHING SHEET
}

\author{
P.G. SIDDHESHWAR* \\ Department of Mathematics, Bangalore University \\ Central College Campus \\ Bangalore - 560 001, INDIA \\ U.S. MAHABALESHWAR \\ Department of Mathematics \\ Government First Grade College for Women \\ Hassan 573 201, INDIA \\ E-mail: ulavathi@gmail.com \\ A. CHAN \\ University of Nottingham (Malaysia Campus) Jalan Broga \\ 43500, Semenyih, Selangor Darul Ehsan, MALAYSIA
}

\begin{abstract}
The paper discusses the boundary layer flow of a weak electrically conducting viscoelastic Walters' liquid B over a nonlinearly stretching sheet subjected to an applied transverse magnetic field, when the liquid far away from the surface is at rest. The stretching is assumed to be a quadratic function of the coordinate along the direction of stretching. An analytical expression is obtained for the stream function and velocity components as a function of the viscoelastic parameter, the Chandrasekhar number and stretching related parameters. The results have possible technological applications in liquid based systems involving stretchable materials.
\end{abstract}

Key words: analytical solution, magnetic field, nonlinear stretching sheet, Walters' liquid B.

\section{Introduction}

Polymer extrusion, drawing of copper wires, continuous stretching of plastic films and artificial fibers, hot rolling, wire drawing, glass-fiber, metal extrusion, and metal spinning are some of the examples where the problem of a stretching sheet arises. Magnetohydrodynamic (MHD) flows are relevant to many practical applications in metallurgy industry, such as the cooling of continuous strips and filaments (sheet) drawn through quiescent liquid, the purification of molten metals from non-metallic inclusions.

Ever since the pioneering works of Sakiadis (1961b), several works have appeared to consider various aspects of the problem (see Siddheshwar and Mahabaleshwar, 2005 and Rajagopal et al., 1987). Pavlov (1974) studied the MHD boundary layer flow of an electrically conducting liquid due to a stretching sheet in the presence of a transverse magnetic field. He neglected the induced magnetic field under the assumption of a small magnetic Reynolds number. The stability of the solution was analyzed by Takhar et al. (1989) and it was found that the magnetic field has a stabilizing effect on three-dimensional disturbances of the Taylor-Görtler type. The core assumption in most of the reported problems is that the stretching is linearly proportional to the axial distance. This is valid provided the stretching process is delicate and slow, leading to the assumption of constant rate of stretching. It is not difficult to see that the above assumption is quite idealistic and impractical. In the strictest sense the stretching has to be nonlinearly proportional to the

\footnotetext{
* To whom correspondence should be addressed
} 
axial distance. The current work is a generalization of the classical work of Andersson (1992) for a linear stretching sheet and presents new results on the problem. In the present paper, as a first step in the general nonlinear modelling exercise, we make use of a simple quadratic stretching model.

\section{Mathematical formulation}

We consider a steady, two-dimensional boundary layer flow of an incompressible and electrically conducting isothermal viscoelastic Walters' liquid B flow due to a quadratically stretching sheet (see Fig.1). A uniform transverse magnetic field $H_{0}$ is applied along the $y$-axis on the weak electrically conducting liquid occupying the half space $y>0$. The magnetic Reynolds number is small and hence the induced magnetic field is negligible as compared to the applied magnetic field. The Walters' liquid B is an approximation for short or rapidly fading memory liquids and is thus an approximation to first order in elasticity. The liquid is at rest and the motion is effected by pulling the sheet on both ends with equal forces parallel to the sheet and with a speed $u$, which varies quadratically with the distance from the slit as $u=\alpha x+\beta x^{2}$. The resulting motion of the otherwise quiescent liquid is thus caused solely by the moving sheet.

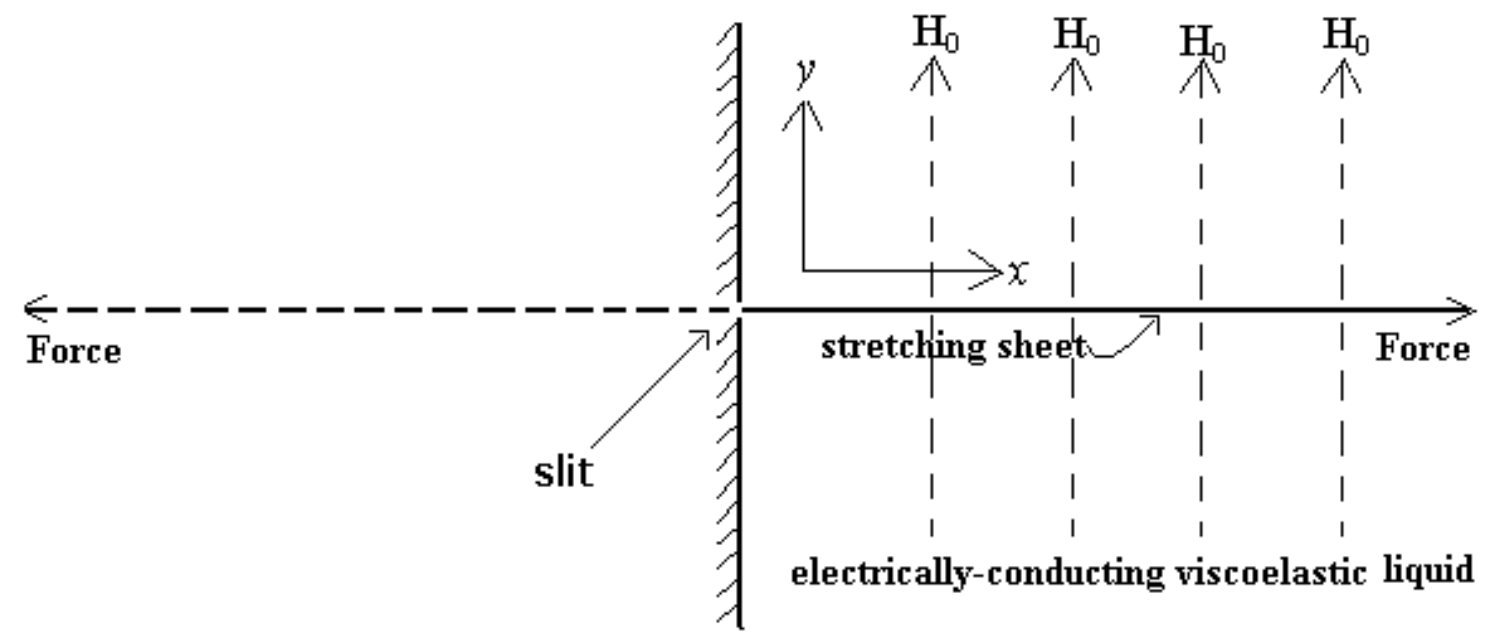

Fig.1. Schematic of the stretching sheet problem.

The steady, two-dimensional conservation of mass and the momentum boundary layer equations for the quadratically stretching sheet problem involving Walters' liquid B are (see Beard and Walters, 1964)

$$
\begin{aligned}
& \frac{\partial u}{\partial x}+\frac{\partial v}{\partial y}=0 \\
& \frac{\partial u}{\partial t}+u \frac{\partial u}{\partial x}+\mathrm{v} \frac{\partial u}{\partial y}=v \frac{\partial^{2} u}{\partial y^{2}}-\left(\frac{\sigma H_{0}^{2}}{\rho}\right) u-k_{0}\left\{u \frac{\partial^{3} u}{\partial x \partial y^{2}}+\mathrm{v} \frac{\partial^{3} u}{\partial y^{3}}+\frac{\partial u}{\partial x} \frac{\partial^{2} u}{\partial y^{2}}-\frac{\partial u}{\partial y} \frac{\partial^{2} u}{\partial x \partial y}\right\}
\end{aligned}
$$

subject to the boundary conditions 


$$
\begin{array}{ll}
u=\alpha x+\beta x^{2} \quad \text { at } \quad y=0, \\
\mathrm{v}=\delta x \quad \text { at } \quad y=0, \\
u=0 \quad \text { as } \quad y \rightarrow \infty .
\end{array}
$$

Here $u$ and $v$ are the components of the liquid velocity in the $x$ and $y$ directions, respectively, $\mu$ is the limiting viscosity at small rates, $H_{0}$ is the applied magnetic field, $v$ is the kinematic viscosity, $\sigma$ is the electrical conductivity and $k_{0}$ is the first moment of the distribution function of relaxation times. Further we assume $\beta$, and thereby $\delta$, is quite small that facilitates the assumption of a weakly two-dimensional flow as considered in the paper.

As pointed out by Vleggaar (1977) in a polymer processing application involving spinning of filaments without blowing, laminar boundary layer occurs over a relatively small length of the zone $0.0-0.5$ $m$ from the die which may be taken as the origin of Fig.1. This is in fact the zone over which the major part of the stretching takes place. In such a process the initial velocity is low (about $0.3 \mathrm{~m} / \mathrm{s}$ ) but not very low enough always to assume linear stretching. Thus a good approximation of the velocity of the sheet is $u=\alpha x+\beta x^{2}$ (at any rate for the first $10-60 \mathrm{~cm}$ of the spinning zone), where $\alpha$ and $\beta$ are the coefficients of the linear and quadratic stretching terms. We have adopted the quadratic stretching model in our problem. The intention of the mathematical formulation as above is twofold:

(i). To seek an analytical solution of the linear stretching sheet and

(ii). To consider the effect of mild perturbations on the classical linear stretching sheet problem.

We now make the equations and boundary conditions dimensionless using the following definition

$$
(X, Y)=\sqrt{\frac{\alpha}{v}}(x, y), \quad(U, V)=\frac{(u, v)}{\sqrt{\alpha v}}, \quad \tau=\beta \sqrt{\frac{v}{\alpha}} t, \quad \beta^{*}=\frac{\beta}{\alpha} \sqrt{\frac{v}{\alpha}}, \quad \delta^{*}=\frac{\delta}{2 \alpha} .
$$

Equations (2.1) and (2.2) take the non-dimensional form as follows

$$
\begin{aligned}
& \frac{\partial U}{\partial X}+\frac{\partial V}{\partial Y}=0 \\
& \beta^{*} \frac{\partial U}{\partial \tau}+U \frac{\partial U}{\partial X}+V \frac{\partial U}{\partial Y}=\frac{\partial^{2} U}{\partial Y^{2}}-k_{l}\left\{U \frac{\partial^{3} U}{\partial X \partial Y^{2}}+V \frac{\partial^{3} U}{\partial Y^{3}}+\frac{\partial U}{\partial X} \frac{\partial^{2} U}{\partial Y^{2}}-\frac{\partial U}{\partial Y} \frac{\partial^{2} U}{\partial X \partial Y}\right\}-\mathrm{Q} U
\end{aligned}
$$

where $\mathrm{Q}=\frac{\sigma H_{0}^{2}}{\rho \alpha}$ is the Chandrasekhar number and $k_{l}=\frac{k_{0} \alpha}{v}$ is the viscoelastic parameter. The parameter $k_{1}$ represents a measure of the relative importance of elastic and viscous effects and can thus be identified with the Weissenberg number. Since we are considering mild perturbation, we have $\beta^{*}<<1$ and hence the time-derivative can be justifiably neglected in Eq.(2.6) do so in the analysis that follows.

Introducing the stream function $\psi(X, Y)$ as

$$
U=\frac{\partial \psi}{\partial Y}, \quad V=-\frac{\partial \psi}{\partial X},
$$


we get from Eq.(2.6) the following

$$
\begin{aligned}
& \frac{\partial^{3} \psi}{\partial Y^{3}}+\frac{\partial \psi}{\partial X} \frac{\partial^{2} \psi}{\partial Y^{2}}-\frac{\partial \psi}{\partial Y} \frac{\partial^{2} \psi}{\partial X \partial Y}=\mathrm{Q} \frac{\partial \psi}{\partial Y}+ \\
& +k_{1}\left\{\frac{\partial \psi}{\partial Y} \frac{\partial^{4} \psi}{\partial X \partial Y^{3}}-\frac{\partial \psi}{\partial X} \frac{\partial^{4} \psi}{\partial Y^{4}}+\frac{\partial^{2} \psi}{\partial X \partial Y} \frac{\partial^{3} \psi}{\partial Y^{3}}-\frac{\partial^{2} \psi}{\partial Y^{2}} \frac{\partial^{3} \psi}{\partial X \partial Y^{2}}\right\}
\end{aligned}
$$

The boundary conditions to be satisfied by $\psi$ can be obtained from Eqs (2.3), (2.4) and (2.7) as

$$
\begin{aligned}
& \frac{\partial \psi}{\partial Y}=X+\beta^{*} X^{2} \quad \text { at } \quad Y=0 \\
& -\frac{\partial \psi}{\partial X}=2 \delta^{*} X \quad \text { at } \quad Y=0 \\
& \frac{\partial \psi}{\partial Y}=0 \quad \text { as } \quad Y \rightarrow \infty
\end{aligned}
$$

The solution to Eq.(2.8), subject to Eqs (2.9), may be taken as

$$
\psi=X f(Y)-\delta^{*} X^{2} f^{\prime}(Y)
$$

where prime denotes differentiation with respect to $Y$. Substituting Eq.(2.10) into Eq.(2.8),

$$
\begin{aligned}
& \left(X-\delta^{*} X^{2} \frac{\partial}{\partial Y}\right)\left(f^{\prime \prime \prime}+f f^{\prime \prime}-f^{\prime 2}\right)+2 \delta^{* 2} X^{3}\left(f f^{\prime \prime \prime}-f^{\prime \prime 2}\right)= \\
& =\left(X-\delta^{*} X^{2} \frac{\partial}{\partial Y}\right)\left(\mathrm{Q} f^{\prime}+k_{l}\left(2 f^{\prime} f^{\prime \prime \prime}-f f^{\prime \prime \prime}-f^{\prime \prime 2}\right)\right)+ \\
& +2 k_{1} \delta^{* 2} X^{3}\left(2 f^{\prime} f^{\prime \prime \prime}-f f^{\prime \prime \prime}-f^{\prime \prime 2}\right) .
\end{aligned}
$$

From Eq.(2.11), one immediately obtains

$$
\begin{aligned}
& f^{\prime \prime \prime}+f f^{\prime \prime}-\left(f^{\prime}\right)^{2}-\mathrm{Q} f^{\prime}-k_{I}\left\{2 f f^{\prime \prime \prime}-f f^{\prime \prime \prime \prime}-\left(f^{\prime \prime}\right)^{2}\right\}=0, \\
& f^{\prime} f^{\prime \prime}-f f^{\prime \prime \prime}=f^{\prime \prime \prime \prime}-\mathrm{Q} f^{\prime \prime}-k_{l}\left\{f^{\prime} f^{\prime \prime \prime \prime}-f f^{\prime \prime \prime \prime \prime}\right\}, \\
& f^{\prime} f^{\prime \prime \prime}-\left(f^{\prime \prime}\right)^{2}+k_{l}\left\{f^{\prime} f^{\prime \prime \prime \prime \prime}-2 f^{\prime \prime} f^{\prime \prime \prime \prime}+\left(f^{\prime \prime \prime}\right)^{2}\right\}=0 .
\end{aligned}
$$

Equation (2.12) turns out redundant as it can be obtained by differentiating Eq.(2.11) once with respect to $Y$. the form

The boundary conditions, for solving Equation (2.11) for $f$ can be obtained from Eqs $(2.9 \mathrm{a}, \mathrm{b}, \mathrm{c})$ in 


$$
\begin{aligned}
& f^{\prime}(0)=1, \quad f^{\prime \prime}(0)=-s, \\
& f(0)=0, \\
& f^{\prime}(\infty)=0, \quad f^{\prime \prime}(\infty)=0
\end{aligned}
$$

where $s=\frac{\beta^{*}}{\delta^{*}}$. One can easily see that Eq.(2.13) is a differential equation for $f^{\prime}(Y)$ and we can also verify that $f^{\prime}(Y)=e^{-s Y}$ is a solution of Eq.(2.13) and this satisfies the derivative boundary conditions in Eqs (2.14). Thus an appropriate solution of Eq.(2.11) is

$$
f(Y)=A+B e^{-s Y}
$$

that satisfies the boundary condition (2.14) provided

$$
A=\frac{\delta^{*}}{\beta^{*}}, \quad B=-\frac{\delta^{*}}{\beta^{*}} \text { and } \quad s=\frac{1}{A} .
$$

We also note that Eq.(2.15) can be a solution of the nonlinear differential Eq.(2.11) if and only if

$$
s=\frac{\beta^{*}}{\delta^{*}}=\sqrt{\frac{1+\mathrm{Q}}{1-k_{l}}} .
$$

We may now write $f(Y)$ from Eqs (2.15) - (2.17) as Dandapat et al. (2004), Andersson (1992)

$$
f(Y)=\frac{1-e^{-s Y}}{s}
$$

It is in place to note here that Dandapat et al. (2004) were the first to obtain this type of solution. Having obtained $f(Y)$ and thereby the stream function $\psi$, we now move on to find the expression for the streamline pattern of the flow in the region around the stretching sheet that can be obtained from Eq.(2.10) as

$$
\psi=X f(Y)-\delta^{*} X^{2} f^{\prime}(Y)=C
$$

where $C$ is a constant. The streamline $\psi=C$ can be written in the functional form as

$$
Y=\frac{1}{s} \operatorname{Ln}\left\{\frac{\frac{X}{s}+\delta^{*} X^{2}}{\frac{X}{s}-C}\right\}
$$

Substituting Eq.(2.10) into Eq.(2.7), we can get the velocity components $U$ and $V$ as 


$$
\begin{aligned}
& U=X f^{\prime}(Y)-\delta^{*} X^{2} f^{\prime \prime}(Y), \\
& V=-f(Y)+2 \delta^{*} X f^{\prime}(Y) .
\end{aligned}
$$

We now discuss the results obtained in the study.

\section{Results and discussion}

The problem of a flexible sheet undergoing quadratic stretching is investigated for the flow it generates in its immediate neighbourhood. The stretching sheet is the sole reason for the liquid flow, and liquid viscoelasticity significantly influences the flow. The flow of the electrically conducting liquid, inhibited by a transverse magnetic field, is studied with the help of streamline patterns and also the axial and transverse velocity distributions. The results are analyzed against the background of the classical linear stretching problem $\left(\delta^{*}=0\right)$ involving Newtonian liquids $\left(k_{1}=0\right.$ and $\left.\mathrm{Q}=0\right)$ (see Crane, 1970). Before we discuss the results of the study, we make some general observations. From Eq.(2.17) it is clear that the $k_{l}$ range of applicability of the solution is $(-\infty, 1)$. This can further be substantiated as follows. Differentiating Eq.(2.11) with respect to $Y$, and subject to condition (2.14), one gets

$$
f^{\prime \prime}(0)=\left(\frac{1-k_{1}}{1+\mathrm{Q}}\right) f^{\prime \prime \prime \prime}(0) \text {. }
$$

From the above equation, we see that $f^{\prime \prime}(0)=0$ for $k_{l}=1$. In conjunction with the condition $f^{\prime \prime}(0)=-s$ in Eq.(2.14a), this would mean

$$
f^{\prime \prime}(0)=-s=0 .
$$

Obviously, for $k_{1}=1$, the quadratic stretching sheet problem ceases to exist. For $k_{l}>1$ we note that $s=\sqrt{\frac{1+\mathrm{Q}}{1-k_{l}}}$ is complex. Hence it stands reiterated that the range of applicability of $k_{l}$ must be $(-\infty, 1)$.

We note that negative values of $k_{1}$ give us the results of a second order liquid and positive values of $k_{1}$ those of a Walters' liquid B model. We now discuss the results of the study on Walters' liquid B followed by those on the second order liquid.

Figure 2 is a plot of the streamline $\psi(X, Y)=1$ for different values of $\delta^{*}$ when $\mathrm{Q}=1$ and $k_{1}=0.2$. Increasing value of $\delta^{*}$ indicates the increasing rate of quadratic stretching. We find from the figure that the increasing rate of stretching restricts the dynamics in the axial direction to regions close to the slit. It is evident from the figures that the Chandrasekhar number Q, viscoelastic parameter $k_{l}$ and the quadratic stretching parameter $\delta^{*}$ work against each other in the lifting of the liquid as we go downstream.

Figure 3 is a plot of various stream lines $\psi(X, Y)=C$ when $\delta^{*}=0.1, \mathrm{Q}=1$ and $k_{1}=0.2$. As is depicted in the figure, at large axial distances the streamlines converge together and are lifted up due to quadratic stretching.

Figure 4 is a plot of the streamline $\psi(X, Y)=1$ for different values of $k_{l}$ and $\delta^{*}=0.1$, when $\mathrm{Q}=1$. It is evident from the aforementioned 3 figures that the viscoelastic parameter $k_{l}$, Chandrasekhar number $\mathrm{Q}$ 
and the quadratic stretching parameter $\delta^{*}$ work against each other in the lifting of the liquid as we go downstream.

Figure 5 is a plot of the streamline $\psi(X, Y)=1$ for different values of $\mathrm{Q}$ and $\delta^{*}=0.1$, when $k_{1}=0.2$. It is evident from the aforementioned 3 figures that the viscoelastic parameter $k_{1}$ and the quadratic stretching parameter $\delta^{*}$ work against each other in the lifting of the liquid as we go downstream.

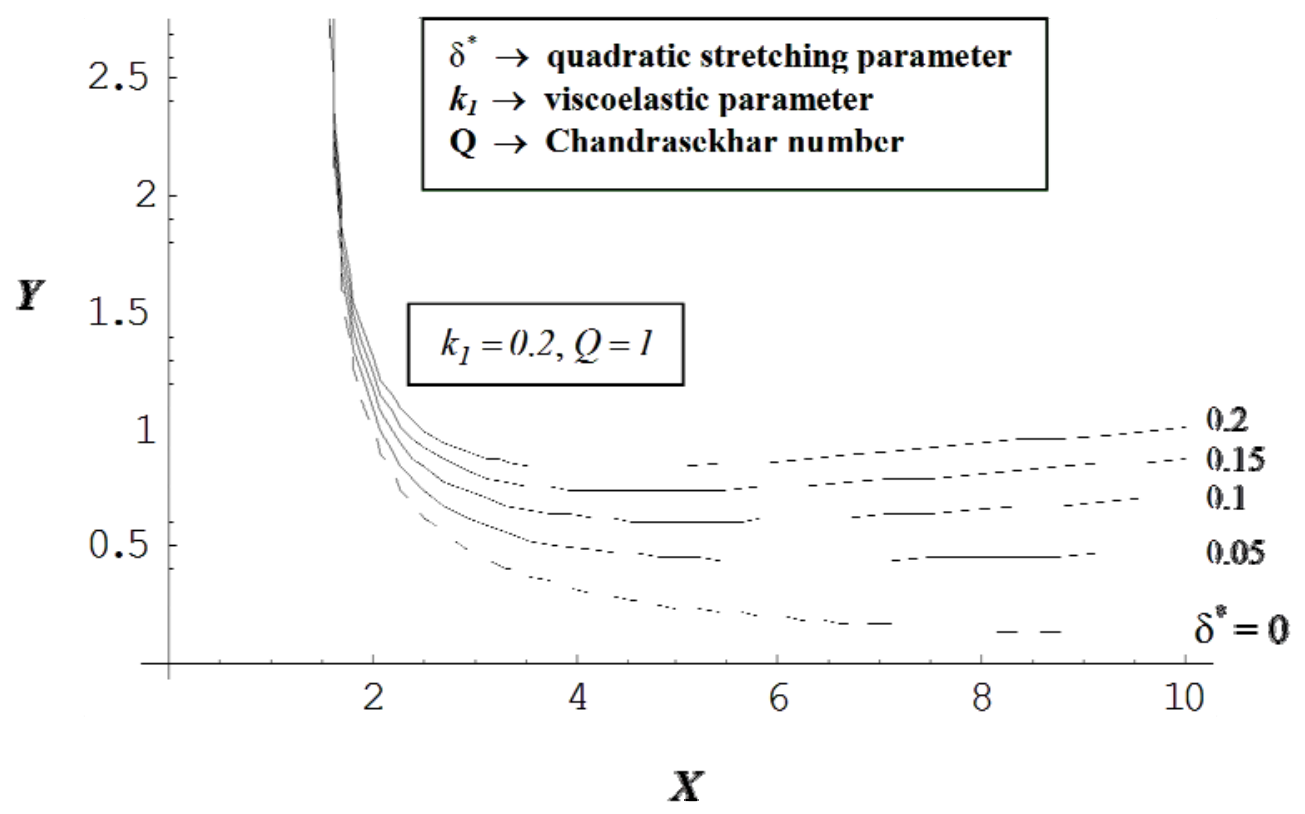

Fig.2. Streamline $\psi(X, Y)=1$ for different values of $\delta^{*}$.

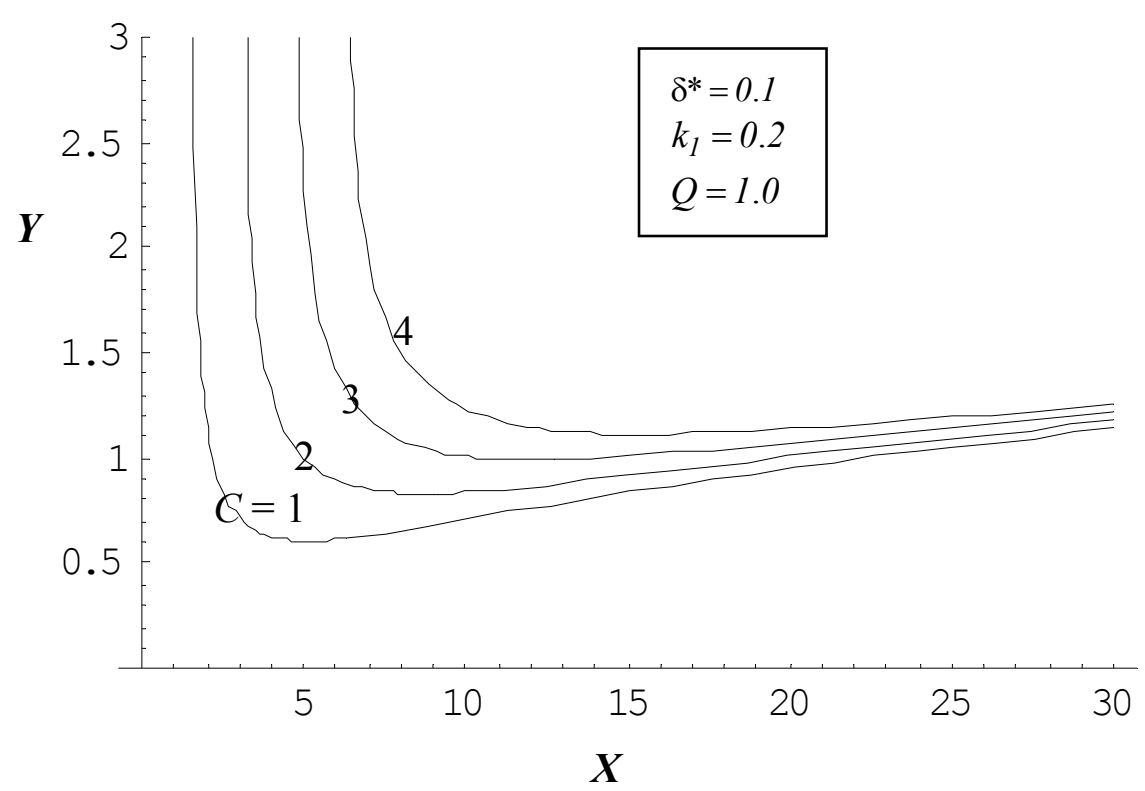

Fig.3. Streamlines $\psi(X, Y)=C$ for different values of $C$. 


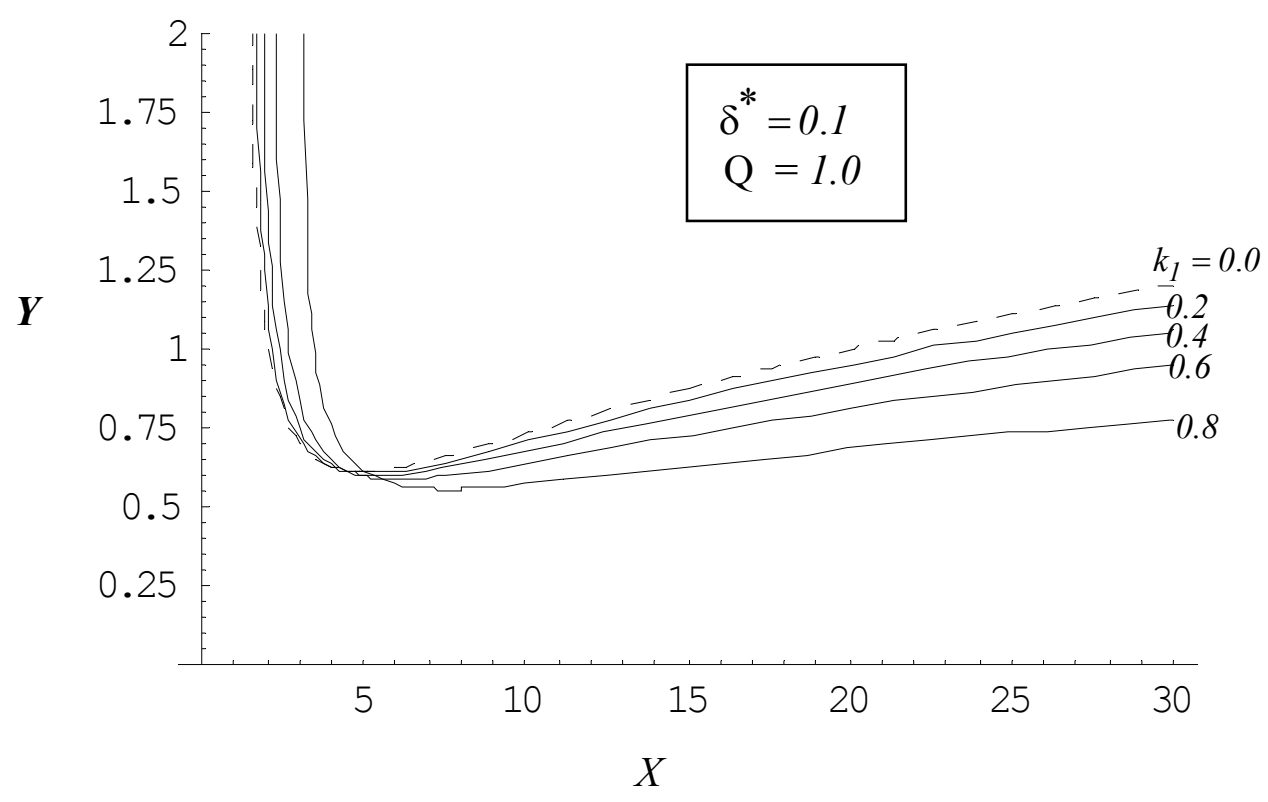

Fig.4. Streamline $\psi^{*}(X, Y)=1$ for different values of $k_{1}$.

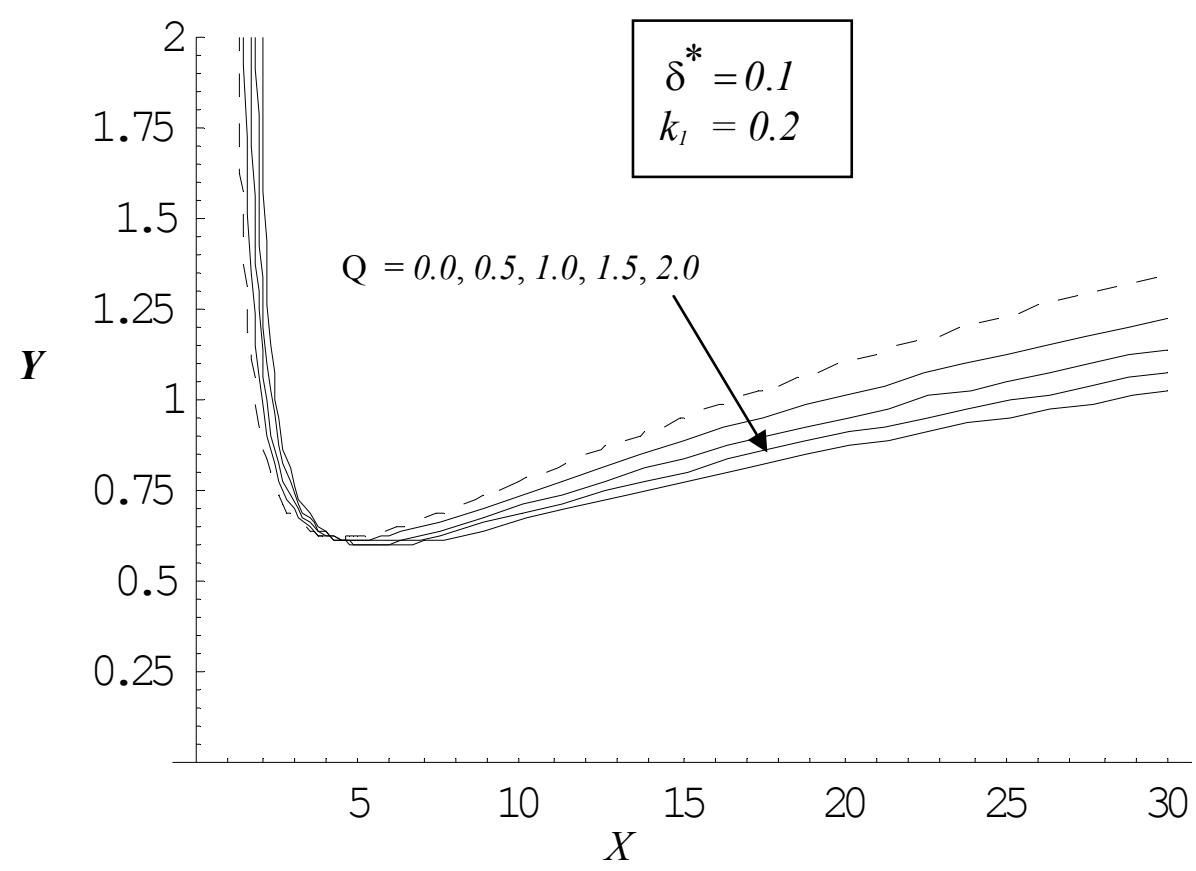

Fig.5. Streamline $\psi^{*}(X, Y)=1$ for different values of $\mathrm{Q}$.

We now discuss the axial and transverse velocity distributions with an observation that

$$
U=X e^{-s Y}+\delta^{*} s X^{2} e^{-s Y} \rightarrow 0 \quad \text { as } \quad Y \rightarrow \infty,
$$

and 


$$
V=-\frac{1-e^{-s Y}}{s}+2 \delta^{*} X e^{-s Y} \rightarrow-\frac{1}{S} \quad \text { as } \quad Y \rightarrow \infty
$$

i.e., the flow outside the boundary layer becomes uniform and is directed perpendicular to the sheet. The boundary layer thickness $\delta_{1}$, defined as the distance from the sheet at which the streamwise velocity $U$ has been reduced to one percent of the velocity $X+\beta^{*} X^{2}$ of the quadratic stretching surface, can be expressed as

$$
\delta_{1}=\frac{\operatorname{Ln}(100)}{s}
$$

Using Eq.(2.17) in Eq.(3.5), we get

$$
\delta_{1}=\sqrt{\frac{1+\mathrm{Q}}{1-k_{1}}} \operatorname{Ln}(100)
$$

Thus, we see that the boundary layer thickness $\delta_{l}$ is coordinate-independent for all permissible values of $k_{1}$. Figure 6 shows the variation of $\delta_{1}$ with $k_{1}$ and Q. We see from the figure that the effect of increasing $k_{1}$ in the range $-\infty<k_{1}<1$ and/or Q is to increase $\delta_{1}$.

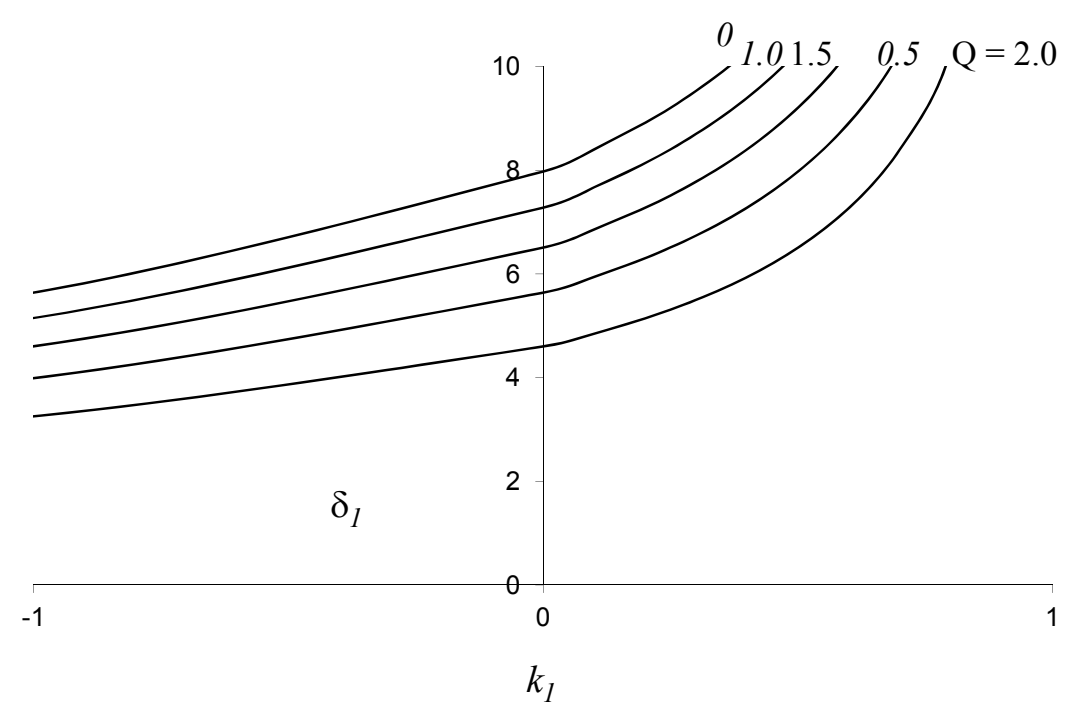

Fig.6. Variation of boundary layer thickness $\delta_{1}$ with $k_{1}$ for different values of Q.

Figures 7-16, that are three-dimensional plots of the velocity components $U(X, Y)$ and $V(X, Y)$, reveal more than the conventional two-dimensional projections on the $U-Y$ and $V-Y$ planes. Figure 7 is a plot of the Crane (1970) profile of the linear stretching problem. One can easily see from the figure that the horizontal and vertical extent of the dynamics on the stretching sheet increases as we go along axial direction.

Figure 8 brings out the effect of the quadratic stretching of the sheet as well as the viscoelasticity of the liquid and the magnetic field. Clearly, both the above effects give rise to an extended dynamic region compared to the linear stretching problem of a Newtonian liquid. Comparing Figs 8 and 9 it is obvious that the quadratic stretching increases the vertical extent of the dynamic region. Comparing Figs 9 and 10 of 
quadratic stretching we find that the effect of viscoelasticity is to initiate lifting of the liquid more closer to the slit compared to that of a Newtonian liquid. On comparing Figs 8 and 10 we conclude that the effect of increasing $\mathrm{Q}$ is to reduce the dynamic region in the vertical extent and increase the same in the axial direction.

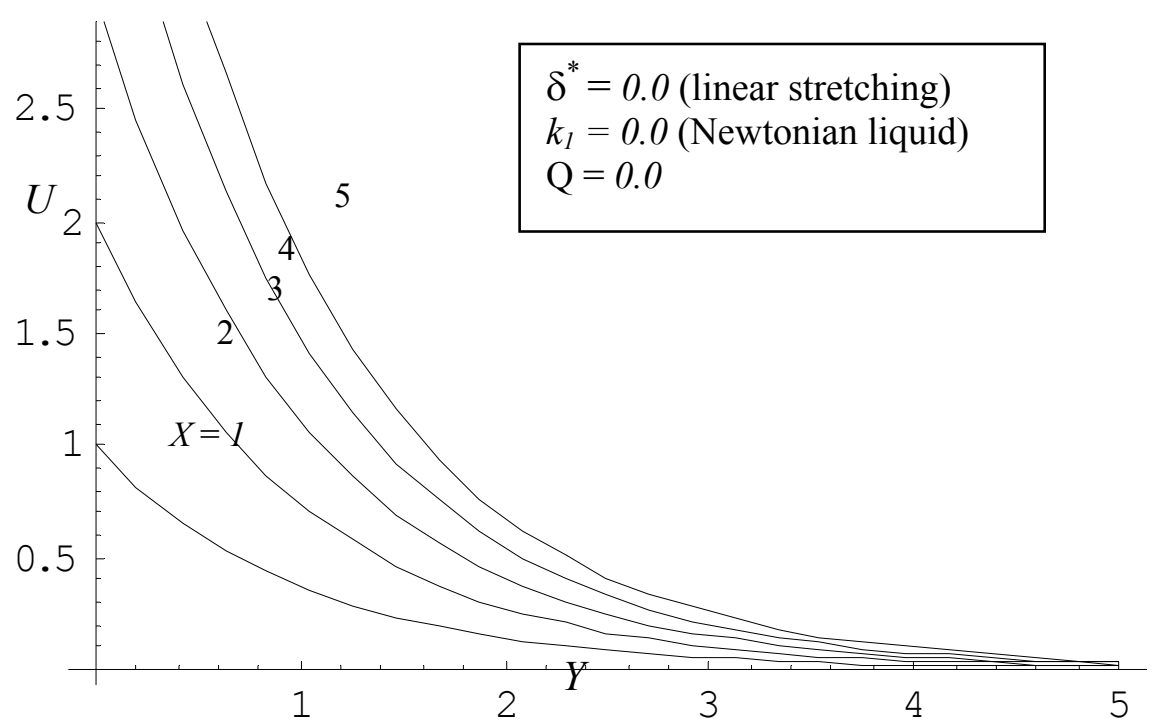

Fig.7. Axial velocity $U(X, Y)$ for the linear stretching sheet problem of Crane (1970).

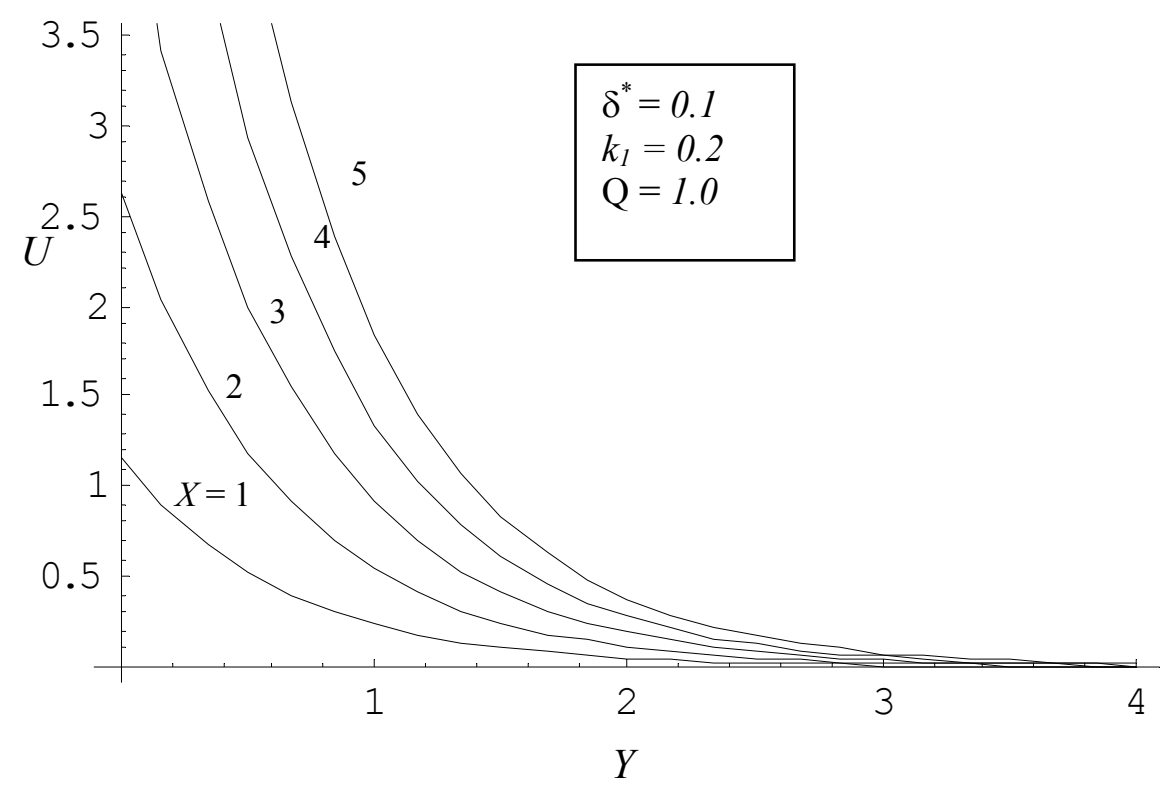

Fig.8. Axial velocity $U(X, Y)$ for the quadratic stretching sheet problem. 


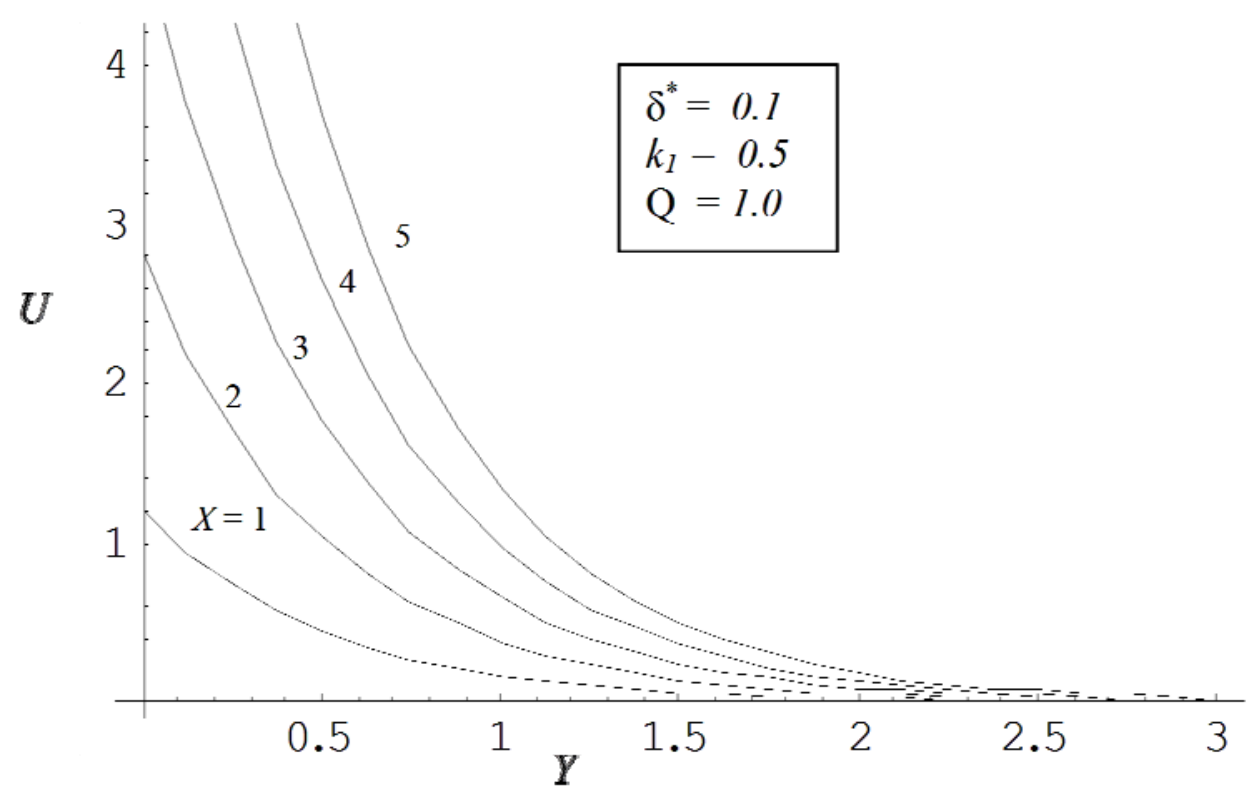

Fig.9. Axial velocity $U(X, Y)$ for the quadratic stretching sheet problem.

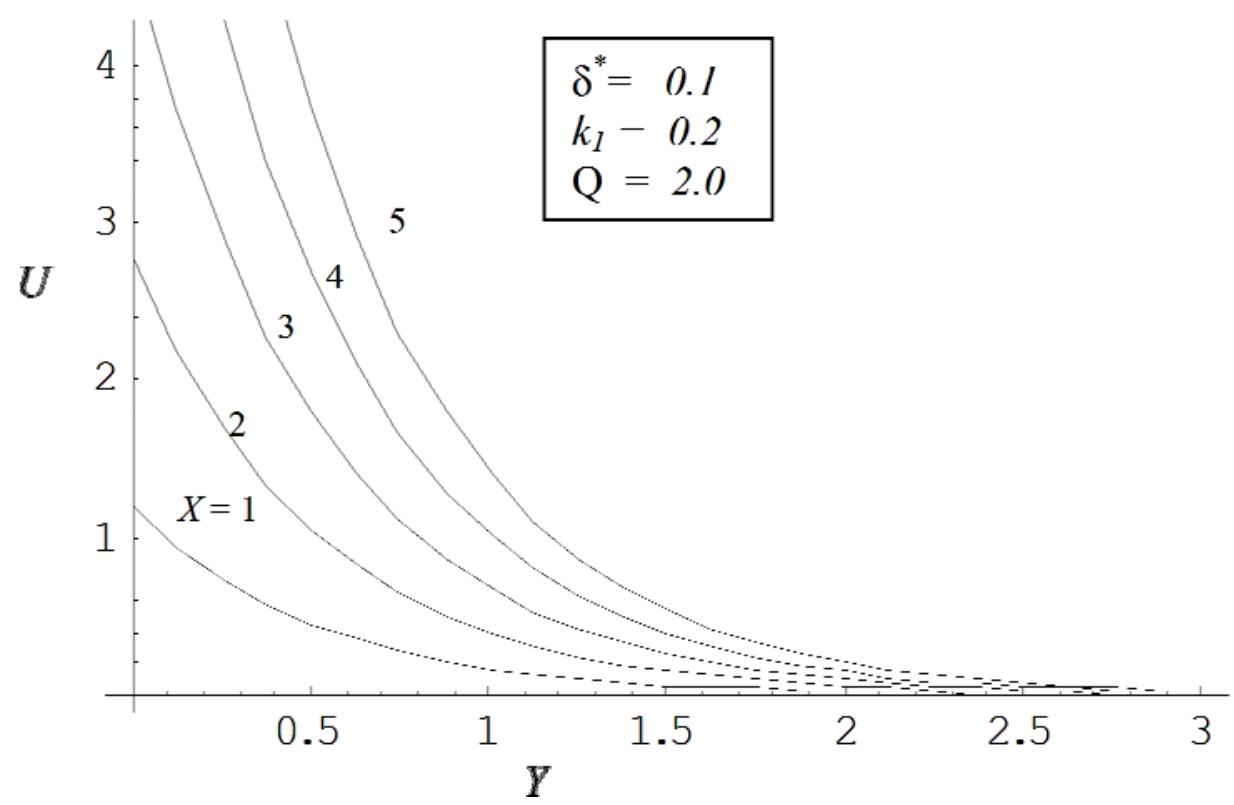

Fig.10. Axial velocity $U(X, Y)$ for the quadratic stretching sheet problem.

The transverse velocity profile brings out the fact that quadratic stretching greatly influences the vertical velocity compared to that in the case of linear stretching. Figure 11 depicts the $X$ - independence of $V$ while Fig. 12 spells out that quadratic stretching induces the $X$ - dependence of the transverse velocity component $V$. Figures 13 and 14 explain the nature of the influence of $k_{l}$ on $V$ for the problem of quadratic stretching. The influence of $k_{l}$ on $V(X, Y)$ is similar to its influence on $U(X, Y)$ and the same is demonstrated by Figs 13 and 14. The influence of quadratic stretching on $U(X, Y)$ and $V(X, Y)$ of a second order liquid shows that the vertical variation is comparatively less than the axial variation. This is demonstrated in Figs 15 and 16 that depict the axial and transverse velocity profiles for the quadratic stretching sheet problem in a 
second-order liquid. The corresponding graphs for a Walters' liquid B model are Figs 8 and 12. It is clear that the lifting is initiated closer to the slit in the case of a Walters' liquid B model compared to the second order liquid. In the case of the latter the flow is more strongly two-dimensional than in the former case. This is clearly seen on comparing Figs 12 and 16.

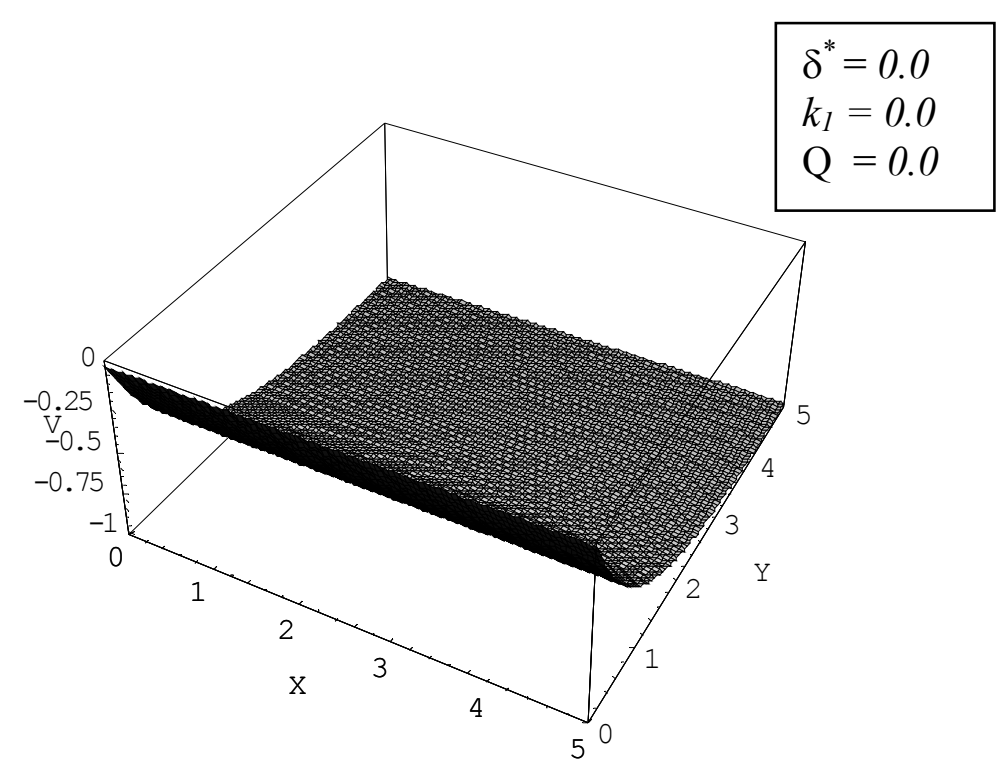

Fig.11. Transverse velocity $V(X, Y)$ for the linear stretching sheet problem of Crane (1970).

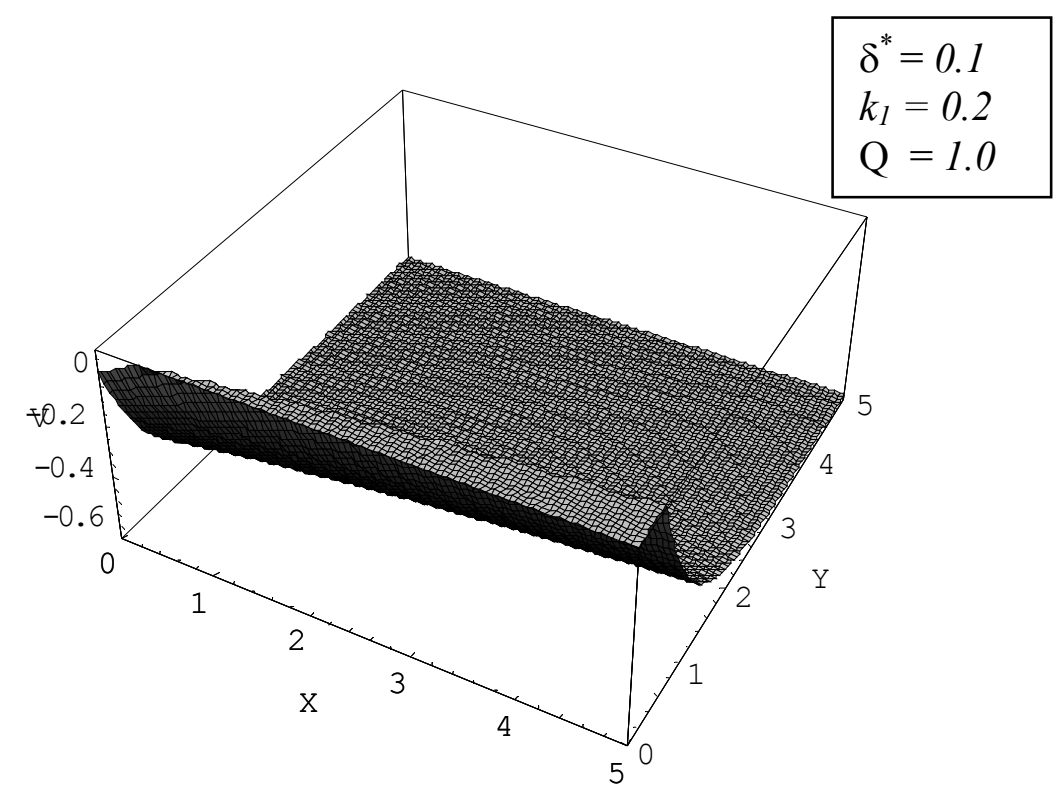

Fig.12. Transverse velocity $V(X, Y)$ for the quadratic stretching sheet problem. 


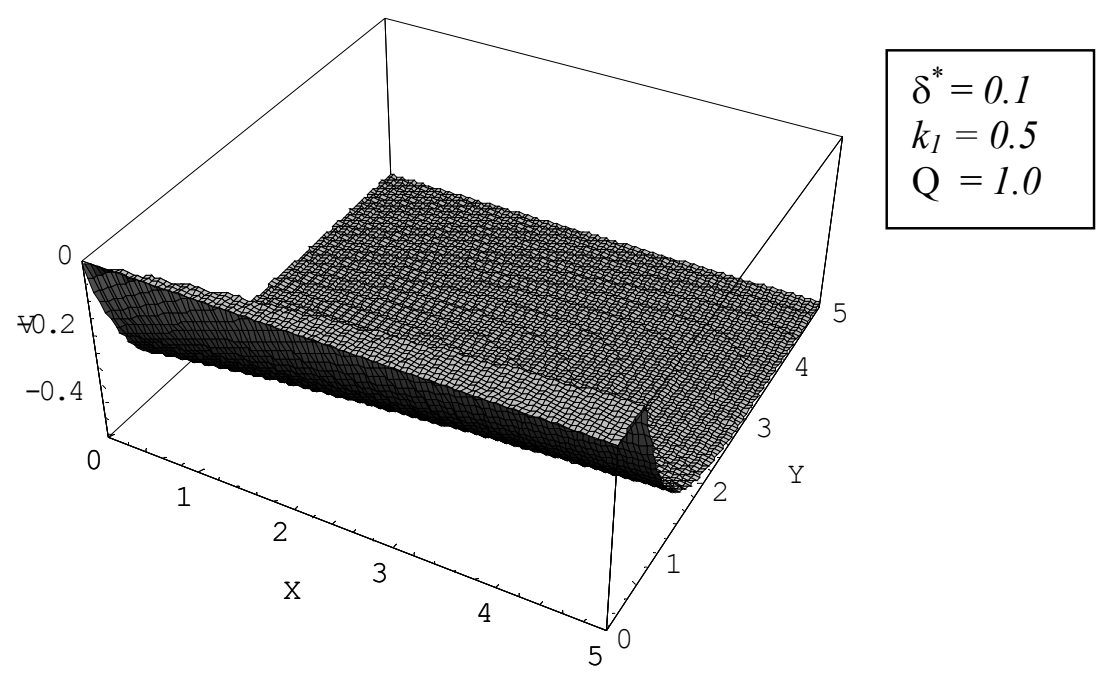

Fig.13. Transverse velocity $V(X, Y)$ for the quadratic stretching sheet problem.

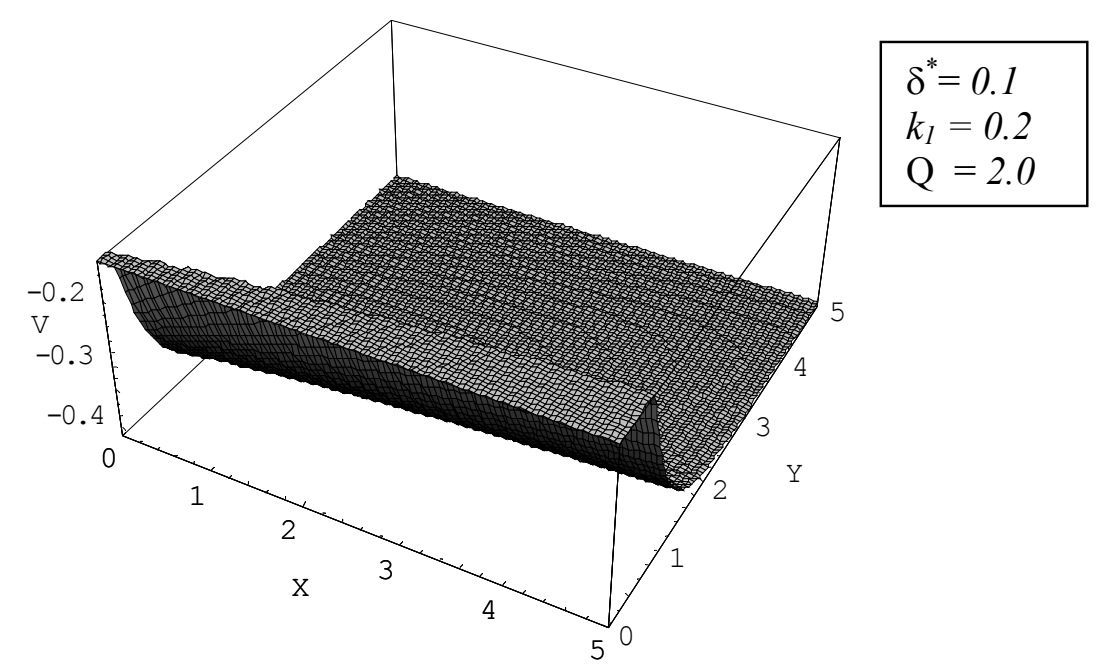

Fig.14. Transverse velocity $V(X, Y)$ for the quadratic stretching sheet problem.

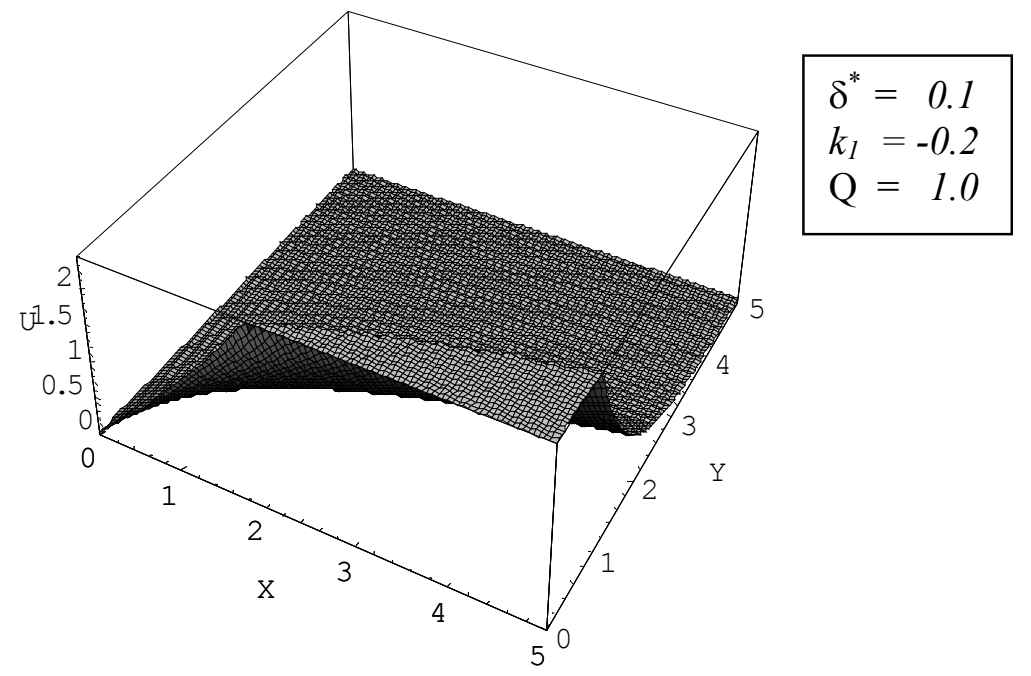

Fig.15. Axial velocity $U(X, Y)$ for the quadratic stretching sheet problem. 


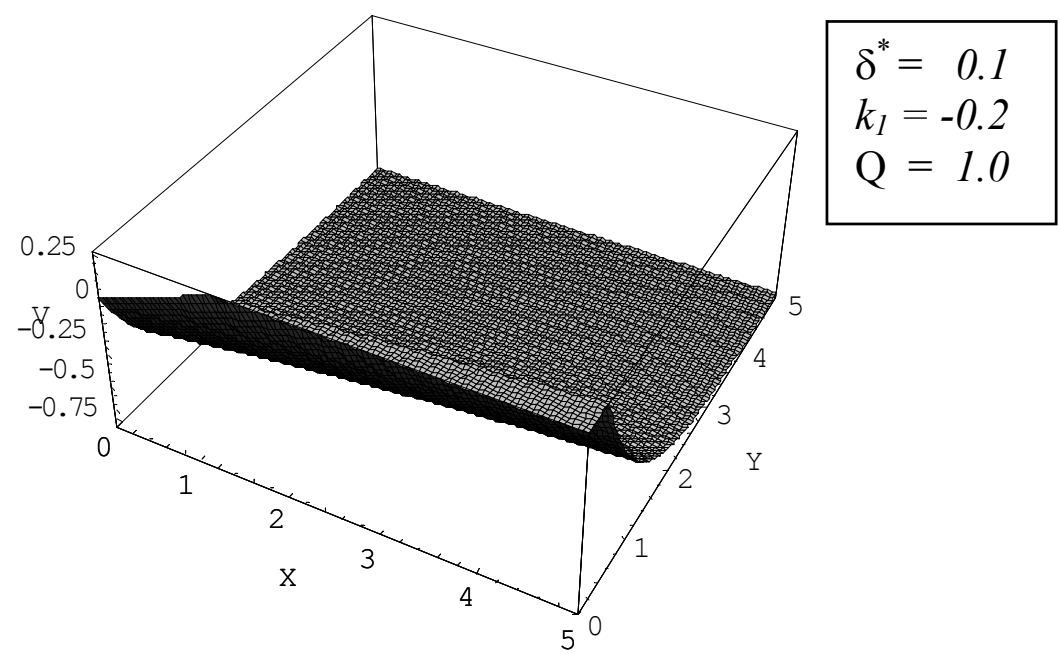

Fig.16. Transverse velocity $V(X, Y)$ for the quadratic stretching sheet problem.

\section{Acknowledgments}

The author Dr. U.S. Mahabaleshwar wishes to thank Bharat Ratna Prof. Dr. C.N.R. Rao F.R.S, Hon'ble Chairman VGST and Dr. S. Anant Raj, Consultant, Department of IT, BT Science and Technlogy, Government of Karnataka, India, for supporting this work under Seed Money to Young Scientists for Research (\# VGST/SMYSR/GRD-304/2013-14).

\section{Nomenclature}

$H_{0}$ - applied uniform vertical magnetic field

$k_{1}-$ viscoelastic parameter

$\mathrm{M}-H_{0} \sqrt{\frac{\sigma}{\alpha \rho}}$ (Hartmann number)

$Q$ - square of $M$

$U$ - dimensionless horizontal velocity

$u$ - dimensional horizontal velocity component

$v$ - dimensional vertical velocity

$V$ - dimensionless vertical velocity

$X$ - dimensionless horizontal coordinate

$x$ - horizontal coordinate

$Y$ - dimensionless vertical coordinate

$y$ - vertical coordinate

$\alpha-$ linear stretching sheet coefficient

$\beta$ - quadratic stretching sheet coefficient

$\delta_{1}-$ boundary layer thickness

$v$ - kinematic viscosity

$\rho$ - density

$\sigma-$ electrical conductivity

$\psi-$ stream function 


\section{References}

Andersson H.I. (1992): MHD flow of a viscoelastic fluid past a stretching surface. - Acta Mech., vol.95, pp.227-230.

Beard D.W. and Walters K. (1964): Elastico-viscous boundary-layer flows. - I. Two- dimensional flow near a stagnation point. - Proc. Camb. Phil. Soc., vol.60, pp.667-674.

Crane L.J. (1970): Flow past a stretching plate. - Z. Angew. Math. Phys., vol.21, pp.645- 647.

Dandapat B.S., Singh S.N. and Singh R.P. (2004): Heat transfer due to permeable stretching wall in presence of transverse magnetic field. - Arch. Mech., vol.56, No.2, pp.127-141.

Pavlov K.B. (1974): Magnetohydrodynamic flow of an incompressible viscous liquid caused by deformation of plane surface. - Magnetnaya Gidrodinamica, vol.4, pp.146-147.

Rajagopal K.R., Na T.Y. and Gupta A.S. (1987): Flow of a viscoelastic fluid over a stretching sheet. - Rheo. Acta, vol.23, pp.213-215.

Sakiadis B.C. (1961b): Boundary-layer behavior on continuous solid surfaces, II. The boundary layer on a continuous flat surface. - A.I.Ch.E. J., vol.7, pp.221-225.

Siddheshwar P.G. and Mahabaleshwar U.S. (2005): Effects of radiation and heat source on MHD flow of a viscoelastic liquid and heat transfer over a stretching sheet. - Int. J. Nonlinear Mech., vol.40, pp.807-820.

Takhar H.S., Ali M.A. and Gupta A.S. (1989): Stability of magnetohydrodynamic flow over a stretching sheet. - In: Liquid metal hydrodynamics (Lielpeteris, J., Moreau, R., eds.), pp.465-471, Dordrecht: Kluwer

Vleggaar J. (1977): Laminar boundary-layer behaviour on continuous accelerating surfaces. - Chem. Eng. Sci., vol.32, No.12, pp.1517-1525.

Received: January 10, 2014

Revised: May 31, 2015 